\title{
Tones encountered with a coannular nozzle and a method for their suppression
}

\author{
K.B.M.Q. Zaman ${ }^{1}$, J.E. Bridges ${ }^{2}$, A. F. Fagan ${ }^{3}$, C. J. Miller ${ }^{4}$ \\ NASA Glenn Research Center \\ Cleveland, $\mathrm{OH} 44135$
}

\begin{abstract}
With multi-stream coannular nozzles, sometimes tones occur that may cause the nozzle to fail noise regulation standards. A two-stream nozzle was studied experimentally and numerically in an attempt to identify the sources of such tones and explore remedies. For the given nozzle configuration, sharp tones occurred in a range of low jet Mach numbers. The tones apparently occurred due to a coupling between vortex shedding from the struts, which held the nozzles and the center-body together, with various duct acoustic modes. A leading edge treatment of the struts is shown to eliminate the tones via disruption of the vortex shedding.
\end{abstract}

\section{Introduction}

There have been numerous previous studies addressing tones occurring with jets and shear layers and their interactions with structures [e.g., 1-5]. The present investigation addresses tones sometimes observed with multi-stream nozzle flows. In fact, the study was prompted by persistent tones encountered in large-scale multi-stream nozzle tests at NASA Glenn Research Center (GRC) [6]. Such tones may make the nozzle fail noise regulation standards and this led to a model-scale experimental investigation. A 0.46-scale model of a two-stream coannular nozzle, that exhibited such tones in the large-scale tests, was fabricated for the investigation. The effort was to understand the origin of such tones and explore possible remedies. This paper reports results from the model-scale experiment.

The flow geometries of complex multi-stream nozzles involve multiple coannular ducts as well as a center-body. Usually, three or four struts, spaced equally in the circumferential direction, hold these coannular elements together. As the flow velocity is increased from zero, distinct tones may occur. With increasing speed a tone may disappear and new tones with different frequencies may appear. As found in this investigation and as explained with the results in the following, one mechanism for the origin of such tones is vortex shedding from the struts. The shedding apparently couples with acoustic resonance modes of the nozzle configuration. The result is an acoustic tone that is spectrally more concentrated than that from vortex shedding alone. A remedy is found. A leading edge treatment of the struts disrupts organized vortex shedding via a disruption of the two-dimensionality of the flow. This may yield a complete elimination of the tones. Key results from the investigation are summarized in this paper.

\footnotetext{
${ }^{1}$ Inlets \& Nozzles Branch, AIAA Associate Fellow.

${ }^{2}$ Acoustics Branch, AIAA Associate Fellow.

${ }^{3}$ Optics \& Photonics Branch, AIAA Associate Fellow.

${ }^{4}$ Acoustics Branch, AIAA Senior Member.
} 


\section{Experimental Facility}

The experiments are conducted in an open jet facility at GRC. A picture of the nozzle mounted on the end of the facility is shown in Fig. 1(a). Unless specifically stated all dimensions are given in inches. A 30" diameter plenum chamber with flow conditioning units is the main component of the facility. Compressed air supplied from one end of the plenum discharges through the nozzle, mounted on the other end, into the ambient of the test chamber. The two-stream nozzle assembly is shown by the drawing in Fig. 1(b), with annotations identifying the various components. It consists of a cylindrical inlet section that is attached to the plenum chamber. This section is of 4.66" diameter and 3.5" in length. The diameter and slope at the junction with the plenum are matched for smooth flow lines. An interested reader may find further description in [7].

With reference to Fig. 1(b), the rest of the two-stream nozzle assembly is bolted to the end of the cylindrical inlet section. The outer nozzle has an exit diameter of 3.46". Compressed air from the single supply splits and passes through the inner and outer passages. The inner flow passes through a removable nozzle having exit diameter of 3.03". Three otherwise identical inner nozzles are used whose lip thickness $\left(t_{\text {lip }}\right)$ is varied as 0.015", 0.030" and 0.050". Items 1 and 2 in Fig. 1(c) represent the inner nozzle with the thinnest and the thickest lip thicknesses, respectively. The "removable inlet' in Fig. 1b is a straight cylindrical section with rounded leading edge. Most of the data pertain to an inlet with length $L=2$ ". Two other inlets of length $L=0.75$ " and 4.75 " (items 3 and 4 in Fig. 1c) are also used in order to vary the (longitudinal) duct acoustic modes and study their impact on the audible tones. The ratio of the flow velocities at the exit of the inner and outer channel is measured to be approximately unity for all hardware at all operating pressures. Items 5 and 6 in Fig. 1(c) are two annular discs that can be placed just upstream of the struts to block either the inner flow or the outer flow.

The inner nozzle and the center-body are held together with respect to the outer shell of the assembly by four struts. The struts are placed in a 'crossed' shape. Thus, both the outer and the inner flows encounter four struts spaced equally in the circumferential direction (Fig. 1b). A sketch of a strut spanning the inner channel is shown in Fig. 1(d). The span $(s)$ is 0.8 " and the chord $(c)$ is $0.625 "$ while the maximum thickness $(t)$ is $0.125 "$. Also shown in this sketch is a leading edge 'treatment' in the form of a cap attached to the strut at mid-span. The cap geometry is described further with the results in the following. Results from numerical analysis of acoustic waves within the nozzle, and associated procedure, are discussed in a separate subsection following the experimental results.

\section{Experimental Results}

Sound pressure level (SPL) spectra measured at a location perpendicular to the nozzle axis, and about 50" away from the exit, are shown in Fig. 2. The spectral traces (0-100kHz range with 1000line analysis) are staggered by $10 \mathrm{~dB}$ and the jet Mach number $\left(M_{j}\right)$ is indicated for each trace. A range of $M_{j}$ from low subsonic to just over unity is covered. These data are for an inner nozzle (Fig. 1b) with lip thickness of 0.030". The spectra display a broadband peak; e.g., at about $25 \mathrm{kHz}$ at $M_{j}=$ 0.256 and $90 \mathrm{kHz}$ at $M_{j}=1.116$. Its frequency continuously increases with increasing $M_{j}$. These peaks are due to vortex shedding from the lip of the inner nozzle, as evident from corresponding non-dimensional value of the frequency. The frequency $\left(f_{s}\right)$ of the peak is plotted in Fig. 3 as Strouhal number based on the lip thickness and jet velocity. Also shown in this figure are corresponding data for two other inner nozzles with lip thicknesses of 0.015" and 0.050". The Strouhal number turns out to be about 0.20 , a value that corresponds to the non-dimensional frequency of von 
Karmán vortex shedding. The rest of the data in this paper pertain to the inner nozzle with 0.030" lip thickness.

In addition to the broadband peak due to vortex shedding from the lip, sharp spikes are noted in the spectra at lower Mach numbers (Fig. 2). These are accompanied by audible tones. The trends of those tones are shown more clearly in Fig. 4 for the same nozzle configuration with spectral traces measured with a narrower frequency range $(0-20 \mathrm{kHz}$ with 1000 -line analysis). As the jet Mach number is increased the frequencies of the tone vary in stages. That is, the frequency remains constant over a range of $M_{j}$ then jumps to a new value and the pattern continues. This is further demonstrated by the tone frequency data in Fig. 5. There are four major stages as marked in the figure. With the calculated velocity within the inner channel near the struts $\left(\approx 0.388 M_{j}\right)$ and the strut thickness, $t=0.125$ " (Fig. 1d), the dimensionless frequency (Strouhal number) in each stage turns out to be about 0.3. This is not too far from the value for Kármán vortex shedding. (The Strouhal number is often found over a range around the expected value of about 0.2 . This is due to the fact that the effective length scale is different from what is used as well as boundary layer effects. In the present case, a thickness smaller than $t$ corresponding to a location closer to the trailing edge is likely to be the length scale, accounting for the higher value of 0.3 that was obtained by using $t$ ). The reader is reminded that, unlike the broadband peaks in Fig. 2 due to shedding from the inner nozzle lip, here we are considering shedding from the trailing edge of the struts. The sharp peaks at lower $M_{j}$, corresponding to the audible tones, are the focus of the present study.

In an effort to understand the nature of the acoustic modes, spectral data were acquired with varying length of the inlet section (Fig. 1b). Corresponding frequency data for the tones are plotted in Fig. 6. Whereas the data in Fig. 5 corresponded to an inlet length $L=2 "$, data for $L=0.75$ " (short) and 4.75" (long) inlets are shown in Fig. 6. Also included are data with the outer flow blocked using the annular disc 6 of Fig. 1(c). It is clear that for any of these parametric variations the tone frequencies and the staging behavior basically remain unchanged. When the inner flow was blocked by disc 5, Fig. 1(c), the tones disappeared. Thus, the source of the tone must be in the inner flow since the tones occur regardless of the presence of the outer flow. Furthermore, somewhat surprisingly, it becomes clear that the length of the inner duct has no impact on the frequencies or the stages of the tones. The duct modes are addressed further with the numerical results in the following. All subsequent data in the paper pertain to the inlet section with length $L=2 "$.

From the discussion of the data in Figs. 4-6 it is apparent that vortex shedding from the struts is the likely origin of the observed tones. However, the fact that the tone frequency remains constant within each stage (Figs. 5,6) indicates that there is a coupling with acoustic resonant frequencies characteristic of the nozzle configuration. When the shedding frequency is close, a particular acoustic resonance mode is excited. The excitation of that mode occurs over a range of the jet Mach number. When the velocity is varied sufficiently the shedding frequency becomes incompatible and the coupling ceases. However, the frequency can now become compatible with another acoustic mode leading to a tone of different frequency. This explains the staging behavior.

It should be mentioned here that various other parametric variations were examined in an effort to understand the origin of the sharp tones. The parameters varied included the lip thickness of the inner nozzle, lip-to-lip distance between the inner and the outer nozzle, a flared and a constricted inlet instead of the straight removable inlet in Fig. 1b, as well as the overall length of the inner duct by varying the length of the removable inlet as already discussed. (When designing the experiment, the 
origin of the tones was suspected to be with the flow near the nozzle exit and its interaction with the duct modes. The struts were not even considered as culprits and hence no provision was made for varying the strut geometry.) For these explorations four conditions were chosen from the four stages of Fig. 5. Corresponding spectral traces at $M_{j}=0.168,0.260,0.345$ and 0.427 are shown in Fig. 7, which display spikes at frequencies $4.5,7.75,9.5$ and $12.38 \mathrm{kHz}$, respectively. For clarity the spikes are marked by the circular symbols. Variation in the lip-to-lip distance was achieved by unscrewing the inner nozzle (that, of course, left a cavity on the inner wall of the inner duct). None of these variations affected the two spectral peaks at the lowest frequencies ( 4.5 and $7.75 \mathrm{kHz}$, Fig. 7). With lipto-lip distance variation the spectral peaks at higher $M_{j}$ were altered with appearance of peaks at other frequencies, probably due to additional tones originating from the inner wall cavity. In general, the tones remained unchanged for variation of the aforementioned parameters and eventually it became apparent that the tones were tied to the struts in the inner duct.

A key result of the study was obtained when the leading edge cap was applied on the inner struts. The tones were completely eliminated as demonstrated in Fig. 8. The caps were cut from a plastic 'edge protector' for car doors. With reference to Fig. 1(d), the dimensions were approximately, $l=$ $0.31 ", h=0.26 "$ and $w=0.64 "$. These were placed centrally on each of the four struts in the inner flow. Experiments showed that placing the caps on the struts in the outer annular flow had no effect on the tones. The tones were suppressed only when the caps were placed on the inner struts. Furthermore, it was found that caps with much smaller span $w=0.3$ " or even 0.1 " were just as effective in eliminating the tones. Apparently, the caps disrupted the two-dimensionality of the flow over the struts and thereby the organized vortex shedding was stopped which in turn led to the elimination of the tones.

A further interesting result was obtained when caps with full span, equal to the span of the struts $(w=0.8 ")$, were tried. With the full-span caps on all four inner struts the tones reappeared albeit at higher Mach numbers. This is shown in Fig. 9. A tone at $4.13 \mathrm{kHz}$ appeared at the highest $M_{j}$. At even higher values of $M_{j}$ two stages of the tone could be detected, as can be seen from the spectra traces of Fig. 10. With the no-cap condition tones were not heard at $M_{j}$ values higher than about 0.43. With the full-caps the occurrence of the tones shifted to a higher $M_{j}$ range and could be heard up to about $M_{j}=0.85$. With the height $h=0.26$ " of the cap (Fig. 1d) and the local velocity in the duct $\left(\approx 0.388 M_{j}\right)$, the frequencies of the two stages are found to correspond to a Strouhal number of about 0.22 . Therefore, it is apparent that with the full-span caps two-dimensionality of the flow is restored that leads to organized vortex shedding which in turn excites the tones. However, since the shedding frequencies are lower due to the larger thickness, for a given acoustic mode the frequency matching occurs at a higher Mach number. This is why the occurrence of the tones has shifted to a range of higher $M_{j}$.

For the full-caps the frequency data are plotted in Fig. 11. Also shown in this figure are the amplitudes of the spectral peaks (in arbitrary scale) to provide an idea about the relative variation of the tone intensity within each stage. Clearly, the amplitude becomes the largest in the middle of each stage. Also, these amplitudes at higher $M_{j}$ with the full-caps turned out to be much larger than those encountered with the no-cap case.

The higher Mach number conditions with the full caps allowed good visualization of the flow using the schlieren technique. Typical visualization pictures for the two stages of Fig. 11 are shown in Fig. 12. The large amplitude tones clearly excite the outer shear layer as evident from the organized 
vortices marked by the downward pointing arrows. Vortex shedding from the lip of the inner nozzle (leading to the broadband peaks, Fig. 2) can be barely discerned as marked by the upward pointing arrows. Unfortunately, neither these pictures nor the experimental results presented so far provide a clear idea about the nature of the acoustic modes within the nozzle that are excited by vortex shedding from the struts. Some insight is gained by the numerical simulation results described next.

\section{Numerical simulation}

The intent of this analysis is to identify duct modes that couple with Strouhal shedding from the struts. A commercial software package, COMSOL Multiphysics ${ }^{\circledR}$ [8], is used to perform the analysis for the 2-stream nozzle configuration. A Computer Aided Design (CAD) geometry (Fig. 1b) is used to create a 3D COMSOL model of the nozzle. The computed domain (Fig. 13) starts upstream of the internal flow split between the core- and bypass-flow paths, and extends downstream to just past the tip of the center plug. The simulation solves the acoustic pressure physics using a finite element formulation on an unstructured mesh. There is no flow in the domain. The upstream boundary has a plane wave radiation condition. The downstream radial and axial surfaces use a spherical wave radiation boundary condition with center on the jet axis and axially at the inner nozzle lip.

Two small panels on either side of one of the struts in the inner flow are used to provide periodic perturbation. The panels are placed near the trailing edge. The 'driven strut' at the top, with the panels marked blue, is identified in Fig. 13. The two sides are driven 180 degrees out of phase in order to simulate the asymmetric vortex shedding. The perturbation pressure amplitude is set arbitrarily at \pm 1 Pascal.

With the perturbation at a given frequency the simulation is performed and the acoustic field in the domain is then interrogated. For each frequency, two metrics for resonance strength are collected: the maximum of the pressure fluctuation amplitude in the domain and the maximum of the root mean square acoustic velocity in the domain. A sweep in frequency is then conducted in steps, e.g., $25 \mathrm{~Hz}, 100 \mathrm{~Hz}$, etc., with larger steps being used at higher perturbation frequencies. This way a spectrum of the response function is constructed that reveals the resonance conditions. Then, for specific resonant frequencies the mode shape, i.e., the nature of the pressure distribution in the domain is studied. As a first step, the mode shape over a cross-sectional plane just downstream of the strut (marked red and identified in Fig. 13) is examined.

The response functions (maximum pressure amplitude within the domain and corresponding maximum r.m.s. velocity) versus the perturbation frequency are shown in Fig. 14. It immediately becomes clear that the simulation approximately captures the tone frequencies as noted in the experiment. The three peaks seen in Fig. 14(b) roughly correspond to three of the peaks seen in Fig. 7 at 4.46, 7.76 and $12.37 \mathrm{kHz}$. The peak at $9.76 \mathrm{kHz}$ (Fig. 7) is missed, however, there is a hint of additional energy around that frequency in Fig. 14(b). Furthermore, the identified resonant frequencies are encouraging in that they exhibit amplification at the non-driven struts, indicating a synchronized Strouhal shedding. As discussed further in the following, the frequency agreement with the experiment is not exact, however, quite satisfactory considering the facts that the simulation is for no-flow and there are experimental uncertainties (approximately $100 \mathrm{~Hz}$ ) due to variations in ambient conditions from day to day. 
The pressure distributions on the monitored plane (Fig. 13) are shown in Fig. 15 for the three dominant resonant frequencies of Fig. 14(b). These frequencies are 4.525, 8.05 and $12.52 \mathrm{kHz}$ in Figs. 15(a), (b) and (c), respectively. Each distribution represents the amplitude in phase with the driven surfaces. Clearly, 'standing wave' patterns have set up among the four struts. A region of either negative or positive pressure exists between a given pair of struts in Fig. 15(a) and the pattern is anti-symmetric across a diameter containing the opposite struts. It appears that the azimuthal spacing of the struts plays a role in dictating the resonance condition. The pattern in Fig. 15(a) corresponds to the tone at the lowest frequency stage (Fig. 5) and thus may be considered as the 'fundamental' in the phenomenon.

The data for resonance at $8.05 \mathrm{kHz}$ (Fig. 15b) exhibit pairs of positive and negative pressure regions within an intra-strut space. Thus, this may be considered as the first harmonic in the phenomenon. The distribution in Fig. 15(c) is also similar but there is an additional radial distribution. This represents a complex higher harmonic. Resonances at many such harmonics are possible. In fact, an eigenmode analysis using the COMSOL solver identified great many eigenfrequencies. For example, 98 modes were found for the given geometry in the 3500-5500 Hz band. Only a selected few of these would be compatible with, and thus excitable by, the asymmetric perturbation from the TE of one of the inner struts. Note that due to prohibitive computational time at high frequencies only onehalf of the cross-section is computed for the case in Fig. 15 (c). Furthermore, the amplified scale in the latter case ( $\pm 1.5 \mathrm{~Pa}$, compared to $\pm 3.5 \mathrm{~Pa}$ in Fig. $15 \mathrm{~b}$ ) made the pressure distribution within the outer annular passage also visible.

A corresponding pressure distribution for the fundamental $(4.525 \mathrm{kHz})$ over the entire domain is shown by six iso-surfaces in Fig. 16(a), for pressures of $\pm 1, \pm 2$ and $\pm 3 \mathrm{~Pa}$. Another perspective of the same domain is shown in Fig. 16(b), skipping the $\pm 2 \mathrm{~Pa}$ iso-surfaces to avoid clutter. These distributions indicate a complex mode shape. It is not a simple longitudinal, helical or radial duct mode. From the $\pm 3 \mathrm{~Pa}$ iso-surfaces, marked in Fig. 16(b), it is apparent that regions of high pressure have formed, against the outer wall of the inner channel, in between pairs of struts. (The walls of the inner and outer channels are made transparent in the figures in order to make the iso-surfaces visible.) The $\pm 1 \mathrm{~Pa}$ iso-surfaces (with lighter red and blue colors, Fig. 16b) touch the 'driven' strut since the driving pressure is of that amplitude. Furthermore, the latter iso-surface touches all the struts demonstrating the synchronized motion in the domain. There must be acoustic feedback among the struts in order to achieve the synchronization of the vortex shedding. Note once again that there is high pressure buildup in the intra-strut spaces. These are locations of pressure antinodes. Therefore, the struts themselves should be locations of pressure nodes and hence anti-nodes for induced velocity. This is indeed found to be the case as demonstrated in Fig. 16(c). High induced velocity near the TE of the struts would be consistent with synchronized vortex shedding in the case with flow.

The high pressure buildup against the inner path outer wall in the intra-strut spaces also indicate reinforcement by reflected waves from the wall, and hence a necessary role of the duct surrounding the struts to establish the standing waves. In other words, such resonance would not be expected if the walls were removed and the strut configuration were left in free space. Except for imposition of a cut-on frequency for the waves to emanate through the nozzle exit, any additional role of the ducts (such as, possible feedback between the struts and the nozzle exit) in establishing the resonances remains unclear at this time. In any case, the standing wave pattern around the struts under the artificial perturbation appears to be the source from which waves propagate in both upstream and 
downstream directions. In practice, with the flow on, the organized vortex shedding substitutes for the artificial perturbation to generate the tones.

Finally, it should be noted that the resonance frequencies found from the simulation were somewhat higher than those seen in the experiment. It is possible this is due to the fact that the simulation involved no-flow. With flow, the feedback from one strut to the next would require longer wavelength, directed upstream at an angle, whose component on the plane of the struts would excite and synchronize with shedding from the adjacent struts. Thus, a simple geometric correction to account for the longer wavelength due to local flow velocity would yield the correct frequencies. Table 1 shows such a correction applied to the predicted frequencies from the no-flow simulation and the resulting agreement with the experimental data.

Table 1 Comparison of predicted frequency with tones observed in the experiment.

\begin{tabular}{|c|c|c|c|c|c|c|}
\hline $\begin{array}{c}M_{j}, \text { Jet } \\
\text { Mach num- } \\
\text { ber }\end{array}$ & $\begin{array}{c}M_{\text {local }} \text {, inner } \\
\text { channel } M \\
\text { at strut loca- } \\
\text { tion }\end{array}$ & $\begin{array}{c}\text { Correction, } \\
\text { Sqrt(1- } M_{l o-} \\
\left.\text { cal }^{2}\right)\end{array}$ & $\begin{array}{c}\text { Freq, } f(\mathrm{~Hz}) \\
\text { Predicted }\end{array}$ & $\begin{array}{c}\text { Freq, } f(\mathrm{~Hz}) \\
\text { Corrected }\end{array}$ & $\begin{array}{c}\text { Freq, } f \\
(\mathrm{~Hz}) \\
\text { Expt }\end{array}$ & $\begin{array}{c}\text { Difference } \\
\text { from } \\
\text { Expt. }\end{array}$ \\
\hline 0.168 & 0.065 & 0.998 & 4575 & 4565 & 4460 & $2.4 \%$ \\
\hline 0.260 & 0.101 & 0.995 & 8095 & 8054 & 7760 & $3.8 \%$ \\
\hline 0.427 & 0.166 & 0.987 & 12690 & 12522 & 12375 & $1.2 \%$ \\
\hline
\end{tabular}

\section{Conclusions}

A two-stream nozzle was studied experimentally and numerically in an attempt to identify the sources of resonant tones. With the given nozzle configuration, sharp tones occurred in a range of low jet Mach numbers. These appeared due to a coupling between vortex shedding from the struts, which held the nozzles and the center-body together, and acoustic resonance modes characteristic of the nozzle configuration. With the present nozzle geometry the source of the tone is determined to be the four struts within the inner channel. A standing wave pattern occurred around those struts whose shape varied and became more complex with increasing frequency. The 'fundamental' involved alternate positive and negative pressure cells in the intra-strut spaces, the pattern being antisymmetric about a diametral plane containing the opposite struts. At higher frequencies, more complex mode shapes occurred involving multiple pressure cells in a given intra-strut space as well as multiple cells occurring in the radial direction. A leading edge treatment of the struts in the inner channel is shown to eliminate the tones. This occurred due to a disruption of the organized vortex shedding. Apart from the nozzle flows considered in this study, conceivably a similar leading edge treatment could be applicable in many other situations, e.g., in wind tunnel tests, where tones induced by similar vortex shedding might be a problem. The remedy is simple and can be included in the original design of the hardware or retrofitted with existing hardware.

\section{Acknowledgement:}

Support from the Commercial Supersonics Technologies (CST) Project of NASA's Advanced Air Vehicles Program is gratefully acknowledged. 


\section{References:}

[1] Rockwell, D. and Naudascher, E., 1979, "Self-sustained oscillations of impinging shear layers", Ann. Rev. of Fluid Mech., vol. 11, pp. 67-93.

[2] Nomoto, H. and Culick, F.E.C., "An experimental investigation of pure tone generation by vortex shedding in a duct”, J. Sound \& Vib., vol. 84, issue 2, pp. 247-252, 1982.

[3] Raman, G. and Srinivasan, K., "Whistles: from toys to industrial devices and applications", Noise Notes, vol. 8, no. 3, July, 2009.

[4] Topalian, V.D. and Freund, J.B., "Acoustic resonance in a model ducted-jet system”, AIAA J., vol. 48, no. 7, pp. 1348-1360, 2010.

[5] Zaman, K.B.M.Q., Clem, M.M. and Fagan, A.F., "Noise from a jet discharged into a duct and its suppression", Int. Journal of Aeroacoustics, vol. 12, no. 3, pp. 189-214, 2013.

[6] Henderson, B.H. and Huff, D.L., The Aeroacoustics of Offset Three-Stream Jets for Future Commercial Supersonic Aircraft, AIAA Paper 2016-2992, 22nd AIAA/CEAS Aeroacoustics Conference, 30 May - 1 June, 2016, Lyon, France.

[7] Zaman, K.B.M.Q., "Increased jet noise due to a "nominally laminar" state of nozzle exit boundary layer", NASA TM 2017-219440, January, 2017.

[8] COMSOL Multiphysics® v. 5.2. www.comsol.com. COMSOL AB, Stockholm, Sweden. 


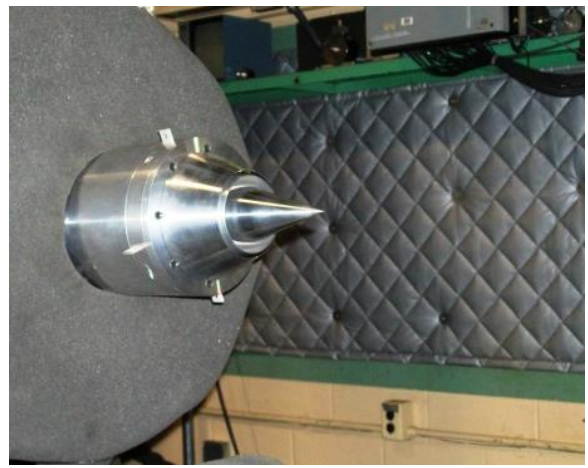

(a)

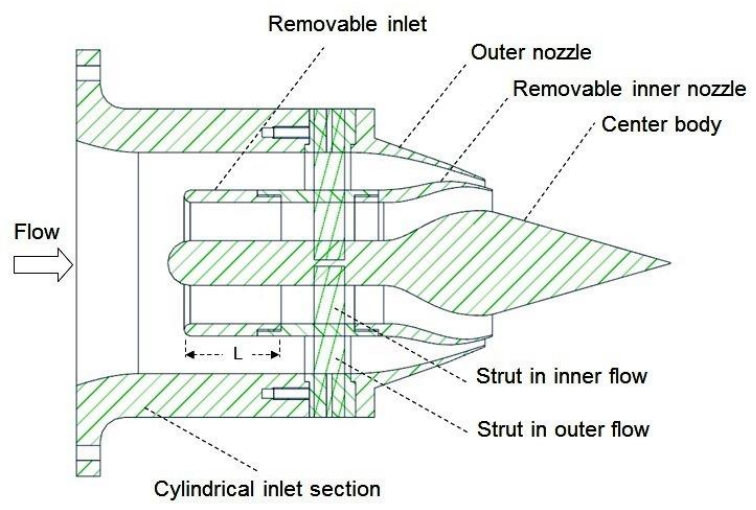

(b)

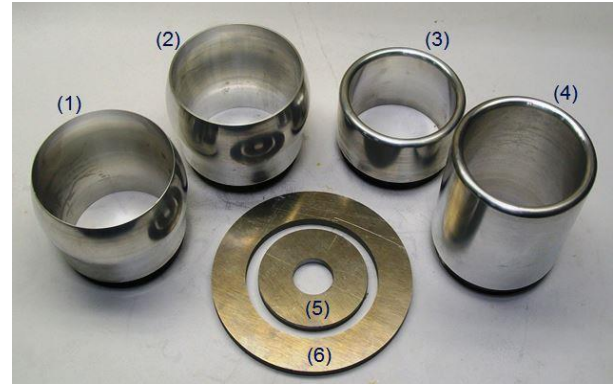

(c)

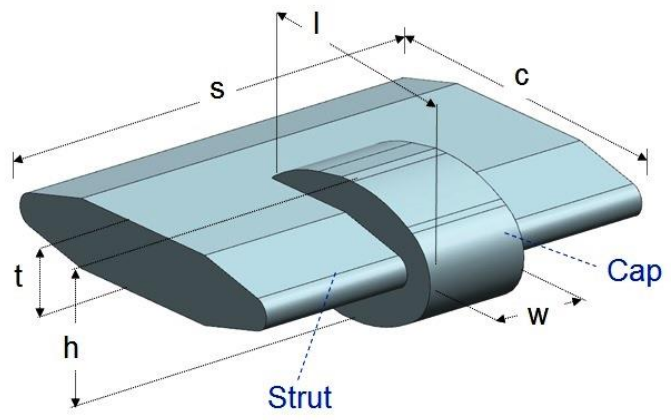

(d)

Fig. 1 Experimental facility. (a) Jet rig, (b) drawing of nozzle assembly, (c) accessories, (d) inner flow strut with leading edge 'cap'.

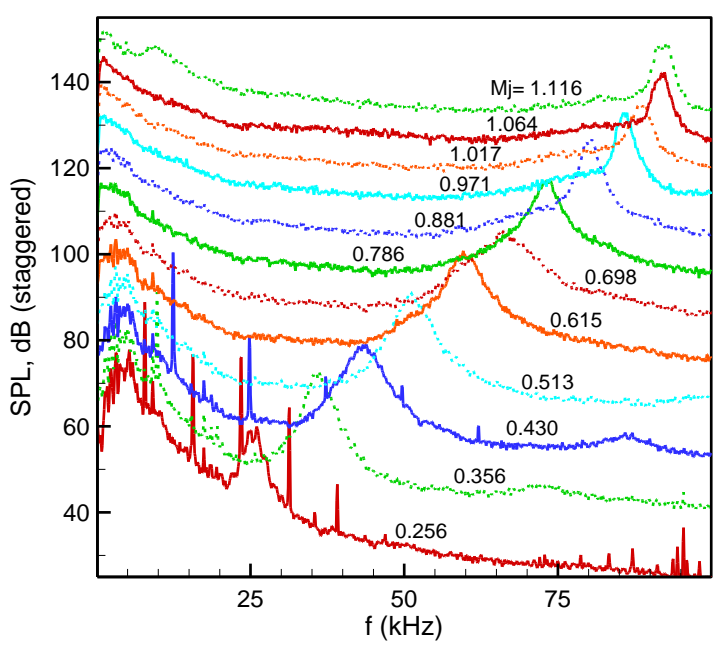

Fig. 2 Sound pressure level spectra at different jet Mach number $\left(M_{j}\right)$ for 0.030 " lip inner nozzle.

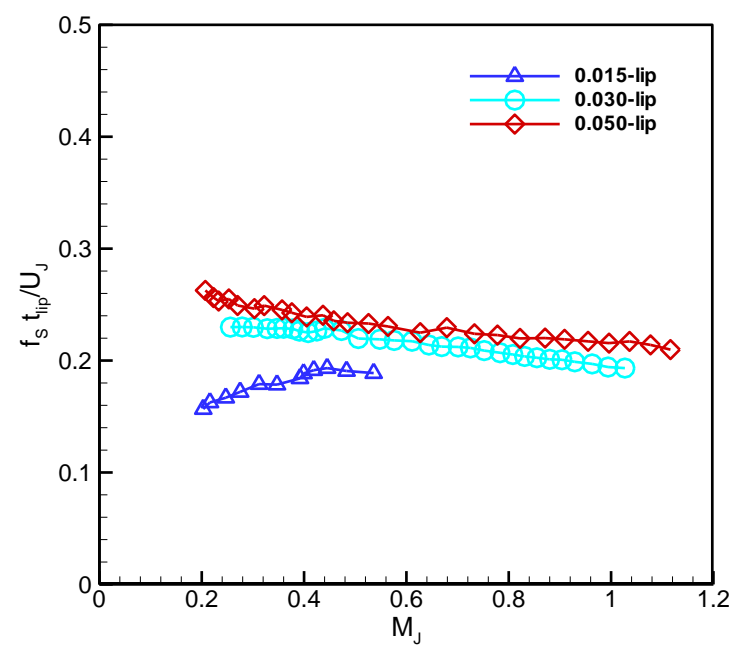

Fig. 3 Strouhal number corresponding to the broadband spectral peaks (Fig. 2) versus $M_{j}$, for three different lip thicknesses of the inner nozzle (Fig. 1b). 


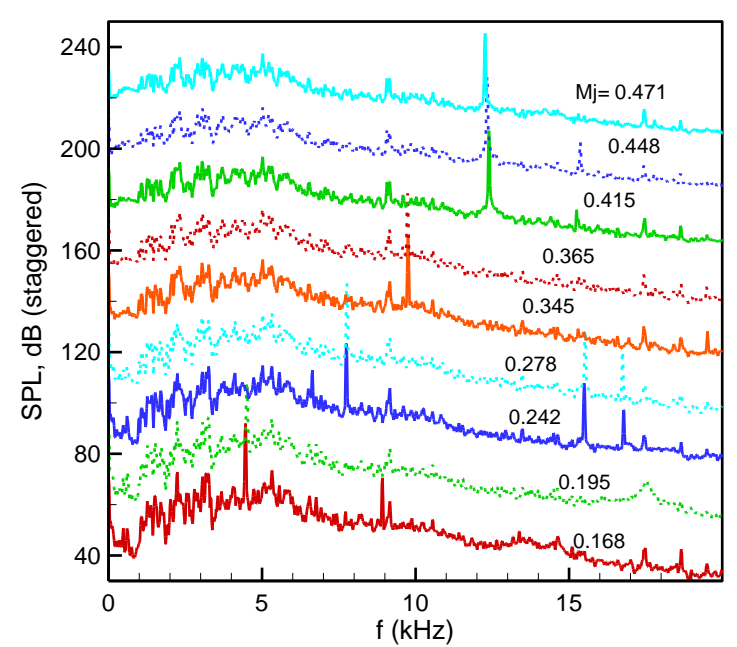

Fig. 4 Sound pressure level spectra at different jet Mach number for the 0.030" lip inner nozzle, in a low $M_{j}$-range with expanded abscissa.

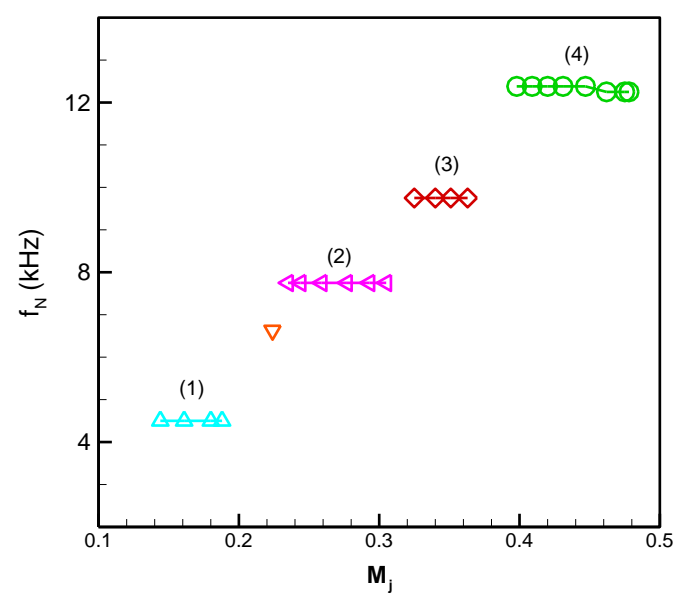

Fig. 5 Tone frequency versus $M_{j}$ for the nozzle case of Fig. 4.

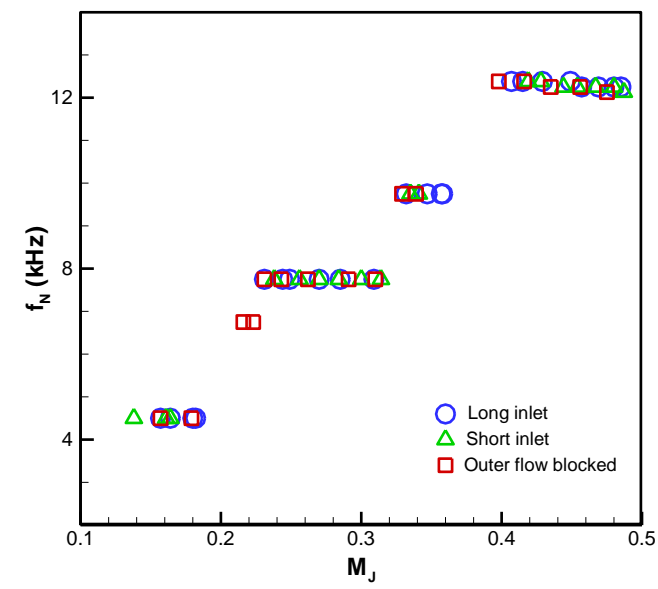

Fig. 6 Tone frequency versus $M_{j}$ with inlets of two different lengths and with a case where the outer flow is blocked.

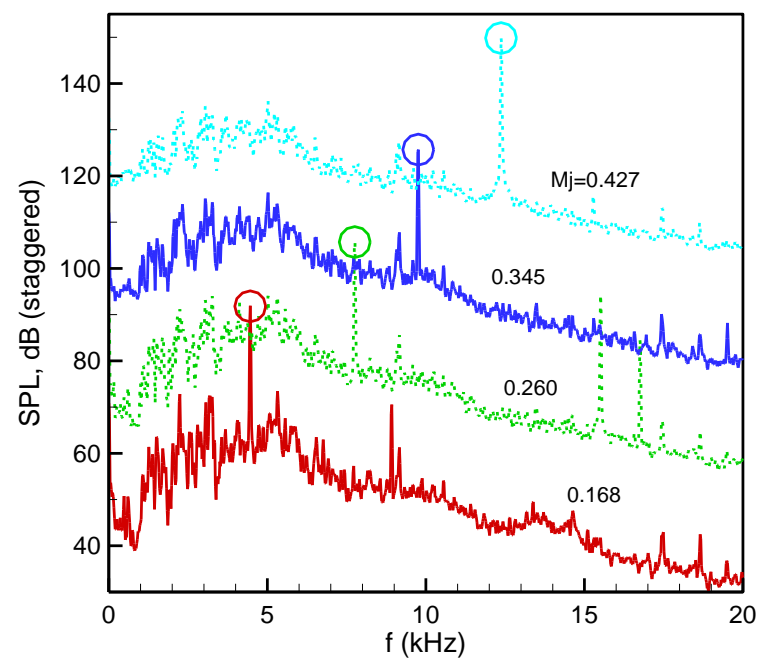

Fig. 7 Sound pressure level spectra at four jet Mach numbers for detailed parametric study. 


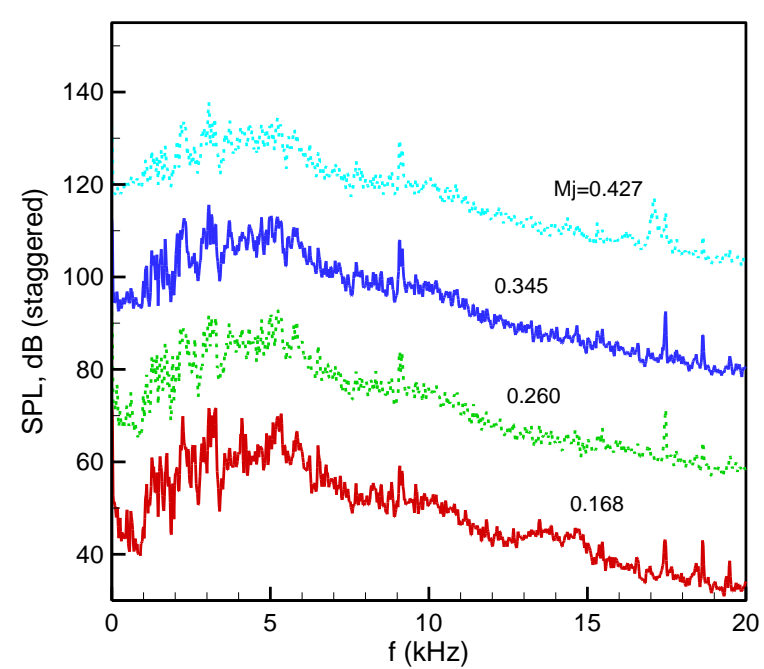

Fig. 8 Sound pressure level spectra at same four $M_{j}$ of Fig. 7 but with semi-span caps on the struts in the inner flow.

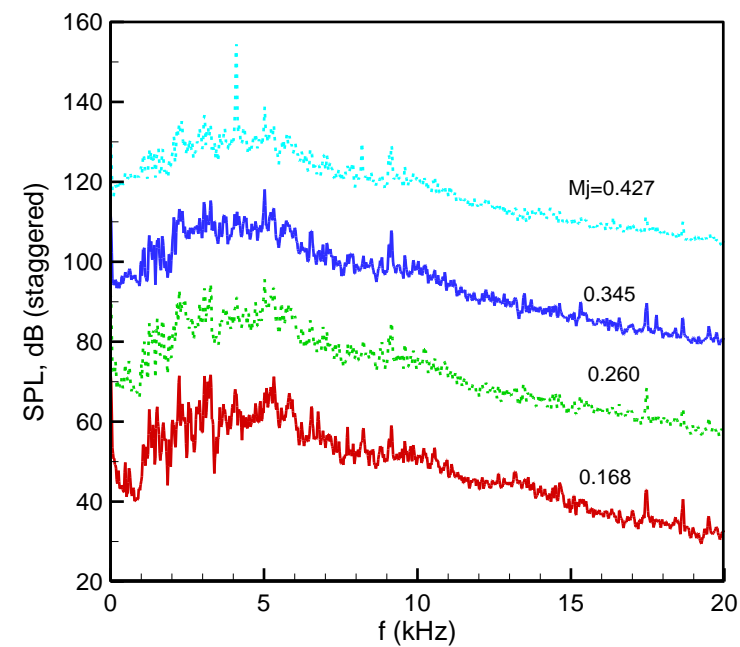

Fig. 9 Sound pressure level spectra at same four $M_{j}$ of Fig. 7 with full-span caps on the struts in the inner flow.

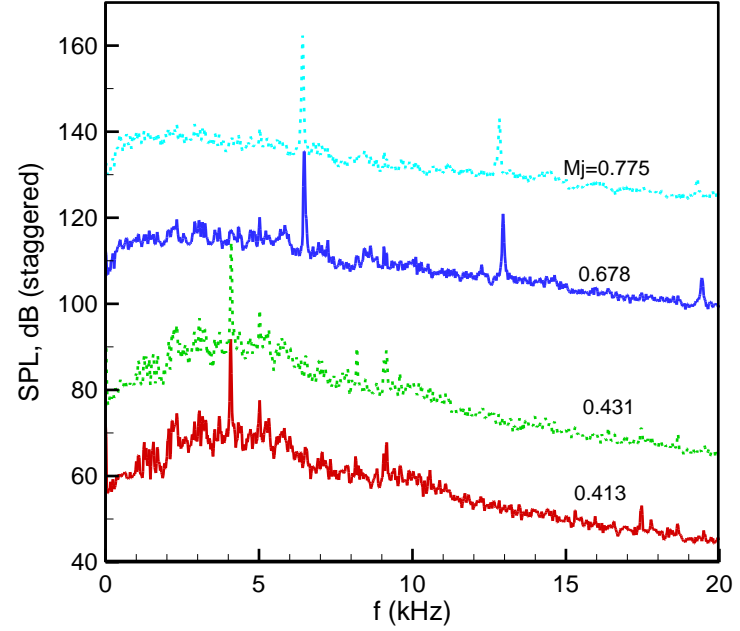

Fig. 10 Sound pressure level spectra with fullspan caps on the struts in the inner flow, at higher values of $M_{j}$.

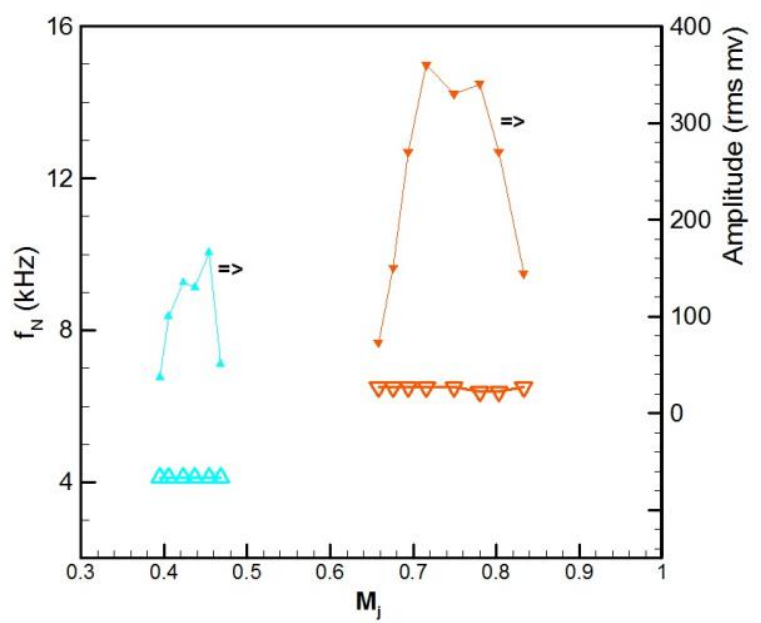

Fig. 11 Tone frequency versus $M_{j}$ with fullspan caps on inner struts. 


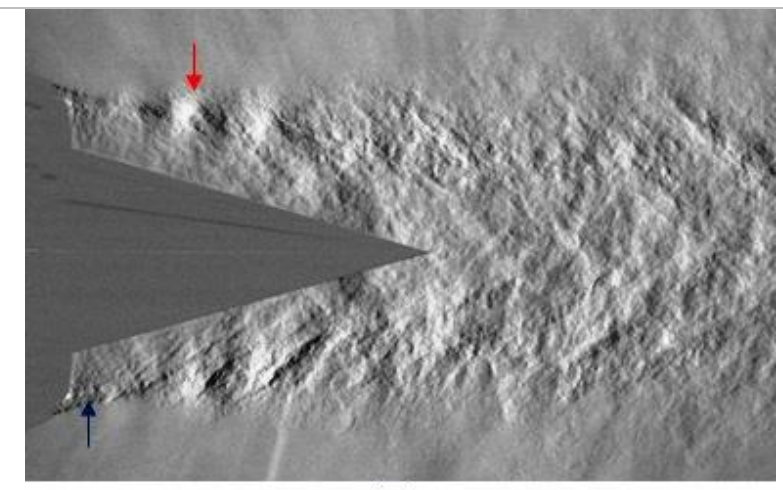

(a)

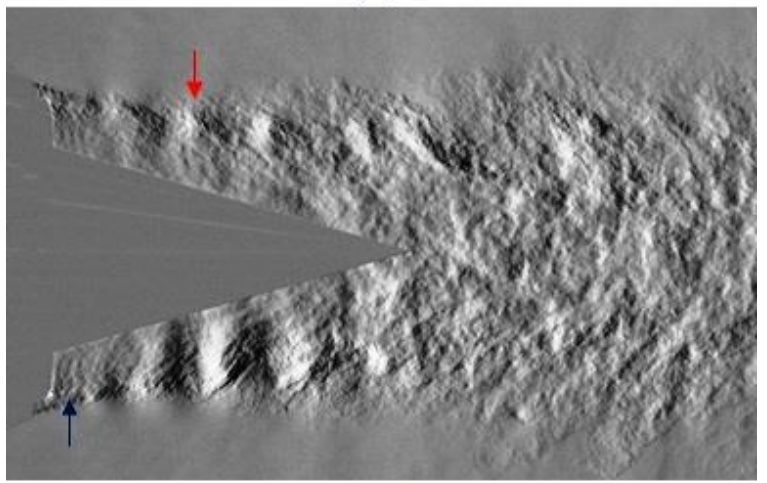

(b)

Fig. 12 Schlieren pictures of the flow; fullspan caps on four inner struts. (a) $M_{j}=0.45$, $f=4.13 \mathrm{kHz}$, (b) $M_{j}=0.67, f=6 \mathrm{kHz}$.

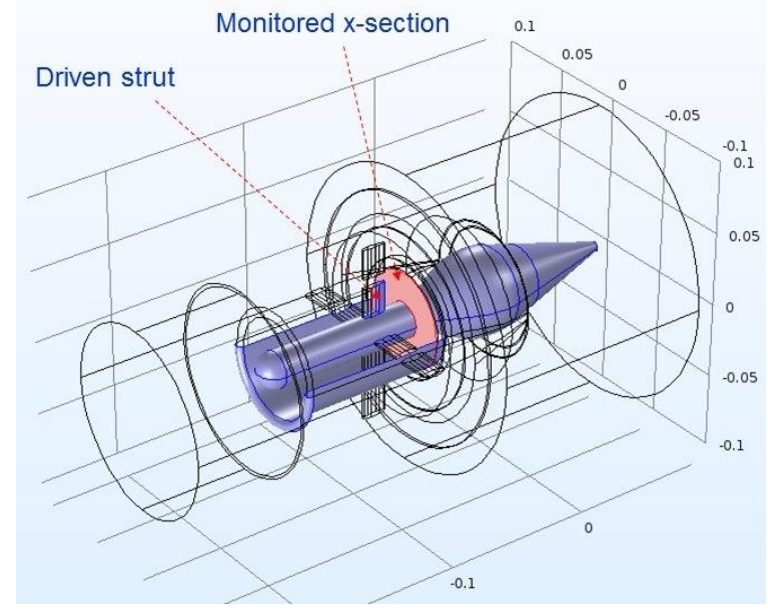

Fig. 13 Computational domain. Monitored plane at the trailing edge of struts is shown in red. Also identified is the driven strut. The coordinate labels in this figure are in meters. (a)

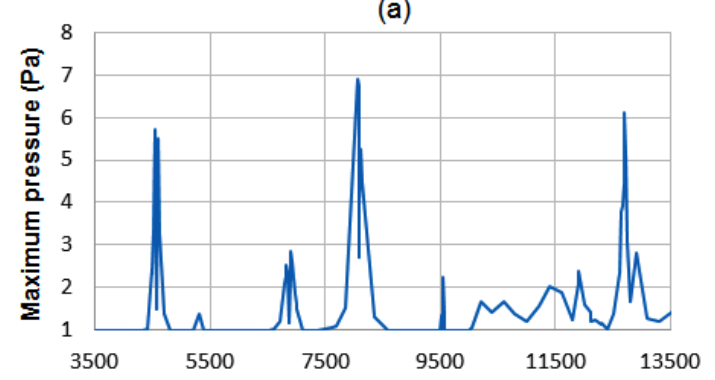

(b)

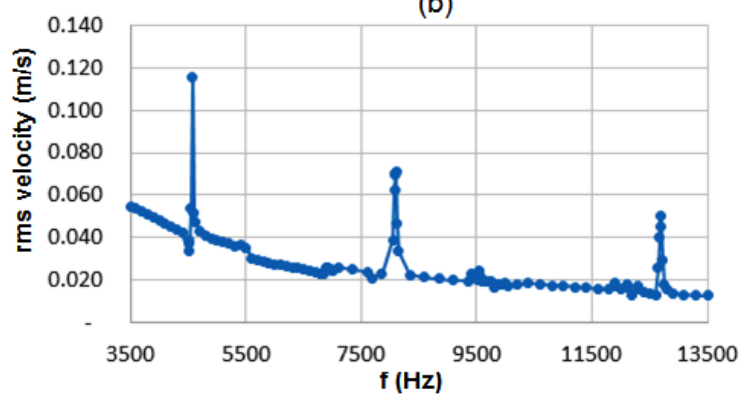

Fig. 14 Maximum of response function within computed domain versus frequency of perturbation. (a) Maximum pressure, (b) maximum r.m.s. velocity. 


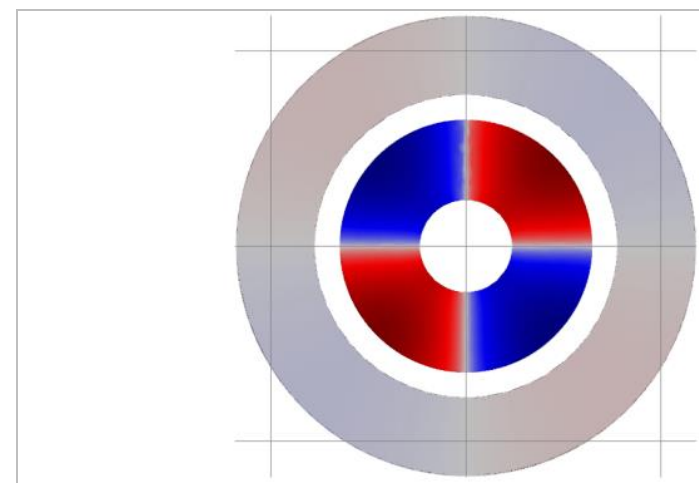

(a)

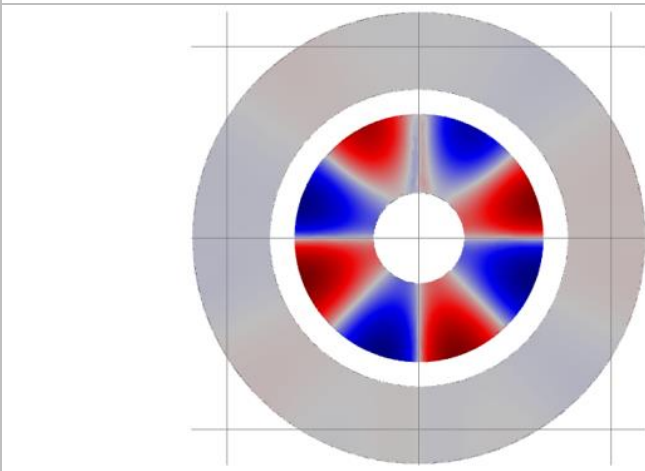

(b)

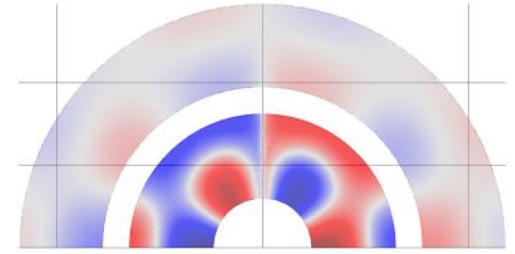

(c)

Fig. 15 Cross-sectional distribution of pressure at the monitored plane (Fig. 13). Frequency and amplitude range are: (a) 4.525 $\mathrm{kHz}, \pm 2.03 \mathrm{~Pa}$, (b) $8.05 \mathrm{kHz}, \pm 3.5 \mathrm{~Pa}$, and (c) $12.52 \mathrm{kHz}, \pm 1.5 \mathrm{~Pa}$.

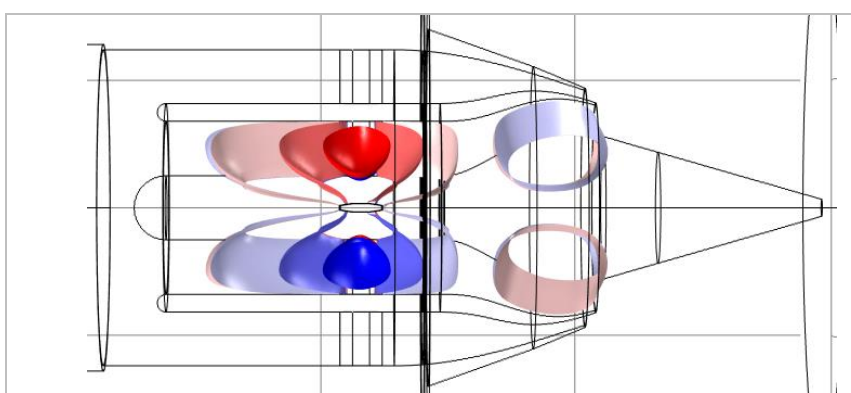

(a)

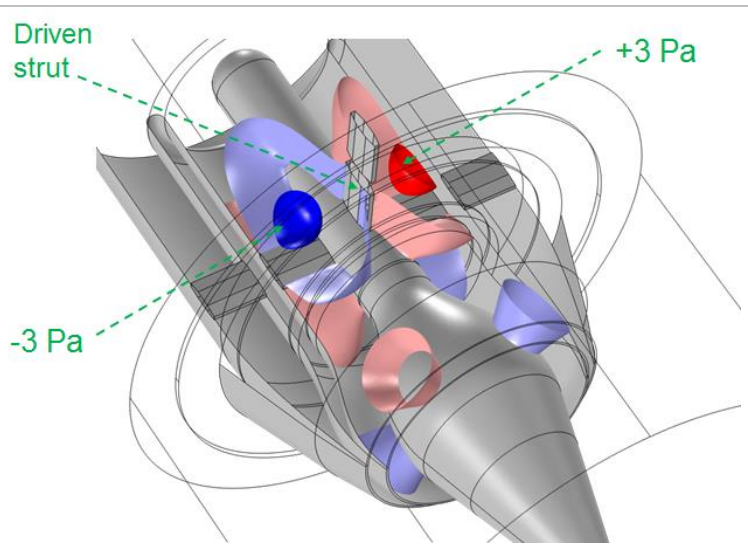

(b)

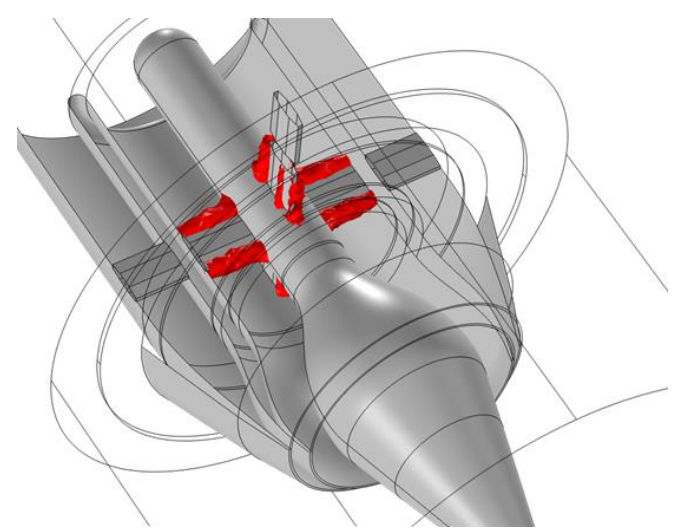

(c)

Fig. 16 Iso-surfaces of pressure and velocity for $f=4.525 \mathrm{kHz}$ over entire domain. (a) Side view with iso-surfaces of pressure at $\pm 1, \pm 2$ and \pm 3 $\mathrm{Pa}$. (b) Oblique view with iso-surfaces of \pm 1 and $\pm 3 \mathrm{~Pa}$. (c) Oblique view with an iso-surface of r.m.s. velocity at $0.006 \mathrm{~m} / \mathrm{s}$. 